\title{
LOS ANIMALES DE LAS RELACIONES INTERRACIONALES, LA UNIÓN EUROPEA Y EL NUEVO ORDEN MUNDIAL
}

\author{
The International Relations' Animals, European \\ Union and the nem Global Order
}

\author{
Javier de Carlos Izquierdo \\ Universidad Complutense \\ E-mail: javierdecarlos@outlook.com \\ Twitter: @javierdecarlos
}

0 Autor

Los animales de las relaciones internacionales son metáforas que nos permiten comprender mejor el mundo actual y, sobre todo, prepararnos para tratar de evitar crisis, emergencias y catástrofes poco previsibles. En este trabajo presentamos los cuatro animales ya conocidos y algunos de sus ejemplos. Ello nos sirve para ilustrar una visión particular de cuál es la situación de la Unión Europea y hacia dónde va el mundo conducido por los principales actores de la escena internacional. Aquí son utilizados los animales de las relaciones internacionales como herramientas de análisis, anunciándonos cuál será el balance de poder de los próximos años, así como algunas estrategias para prepararnos ante estos cambios.

The International Relations' Animals are metaphors that allow ourselves a better understanding of today world and even these animals can help us to avoid catastrophic events or crisis difficult to be foreseen or predicted. This paper shows us some animals that we introduced before in Comillas Journal of International Relations (12). Now we discuss, helping with our animals, about European Union and International balance of power. Hence International Relations' Animals are assessment tools for protect us from New Global Order power some strategies for the next years.

Animales de las relaciones internacionales; cisnes negros; elefantes negros; medusas negras; rinocerontes grises; Unión Europea; nuevo orden mundial.

Key words

International Relations Animals; black swans; black elephants; black jellyfish; grey rhinos; European Union; New Global Order. 


\section{Introducción}

En los últimos años se han empleado algunos animales como metáfora para entender mejor los conflictos del mundo actual. Estas metáforas permiten reflexionar a los economistas, científicos o analistas políticos para tratar de anticiparse a los hechos que están por llegar. El siglo XXI es diferente a la centuria pasada, el proceso de globalización continuado y sostenido ha producido cambios acelerados que provocan nuevas crisis y en ocasiones la inestabilidad internacional. Las metáforas aquí analizadas pueden ser utilizadas como herramientas para tratar de anticiparse a estas situaciones o llegado el caso superarlas con mayor facilidad. Hoy en día, el orden internacional resultado de la Guerra Fría ha desaparecido, nos encontramos en una etapa de transición en la que el sistema que regula las relaciones internacionales también está siendo sustituido por otro nuevo. En este trabajo tratamos de alumbrar estos hechos y conocer algunas implicaciones para la Unión Europea, utilizando los nuevos animales de las relaciones internacionales. Entre estos animales cabe destacar a los cisnes negros, los elefantes negros, las medusas negras y los rinocerontes grises, todos ellos son herramientas muy provechosas para el estudio de las relaciones internacionales. Más adelante resumimos estas metáforas, aunque ya han sido tratadas con cierto detalle en Comillas Journal of International Relations (Carlos, 2018a).

\section{Las relaciones internacionales y sus animales}

En el año 2007 Nassim Taleb definió un cisne negro como un acontecimiento atípico, que produce consecuencias traumáticas y que no se puede prever (Taleb, 2007, XVIII-XIX). Taleb había avisado sobre la llegada de la crisis financiera de 2007 a pesar de que los indicadores económicos no la anunciaban. Un ejemplo clásico de cisne negro es el atentado de las Torres Gemelas de Nueva York en 2001. Pero Taleb también nos señala otros ejemplos de cisnes negros como la II Guerra Mundial, la caída del Muro de Berlín, o la expansión del fundamentalismo islámico. Del mismo modo que cuando vemos un cisne esperamos que este sea blanco, los cisnes negros son poco frecuentes, inesperados e imprevisibles.

Figura 1. El cisne negro

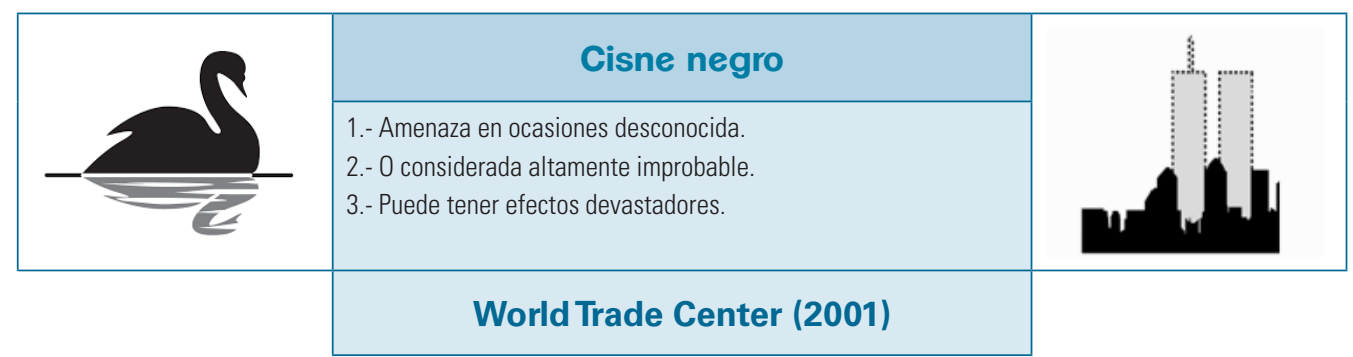

En realidad, un cisne negro es un acontecimiento sorprendente en relación a la información que se conoce, pero a posteriori siempre estos sucesos son considerados previsibles. Sobre todo la metáfora del cisne negro es una crítica a los análisis estadísticos y también a los programas de gestión de riesgos informáticos, ya que estas herramientas analizan fundamentalmente tendencias y no los aspectos atípicos o irregulares que, a veces, son los más importantes. Por ello, para evitar que los cisnes negros nos sorprendan debemos estar atentos a los acontecimientos 
extraordinarios, aunque no parezcan significativos, porque si nos centramos solamente en lo habitual nuestro conocimiento será irrelevante y no estaremos prevenidos contra los sucesos más peligrosos y menos deseados.

Todos sabemos que los elefantes y los cisnes negros son animales distintos, lo mismo ocurre en las relaciones internacionales. Un elefante negro hace alusión a un problema que es visible para todos y que tendrá enormes consecuencias, pero a pesar de ello nadie se interesa por él. Este símil fue utilizado por Adam Sweidan en 2014 al referirse al cambio climático.

Figura 2. El elefante negro

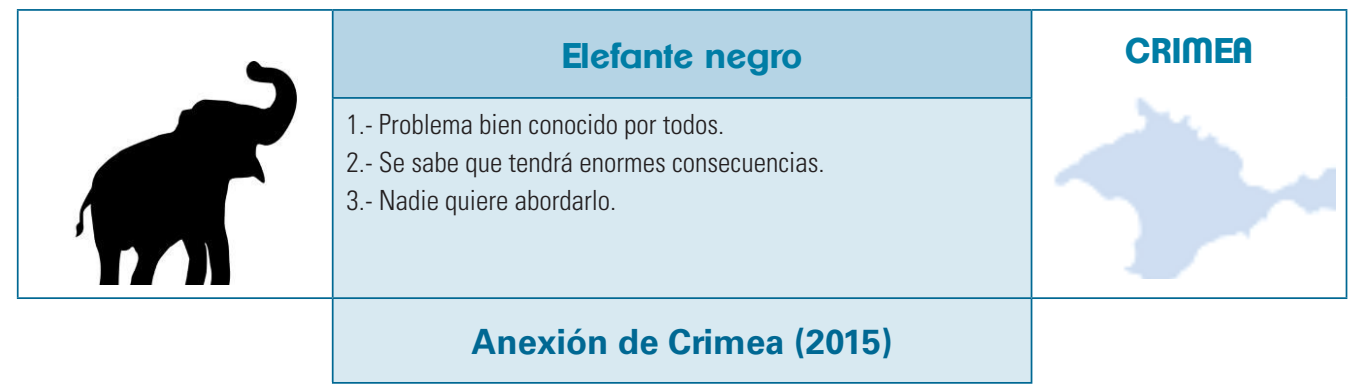

Un elefante negro es un problema real e importante que es ignorado por todos a pesar de que se conocen sus posibles consecuencias fatales. Situaciones de todos conocidas y a las que se tardó tiempo en poner el cascabel al gato han sido la crisis de Ucrania o incluso la crisis migratoria en Europa.

El cambio climático y la elevación de la temperatura del agua en los océanos han provocado el aumento de la población de las medusas, creando problemas ecológicos de todo tipo, incluso en los reactores de las centrales nucleares. La proliferación de estos animales se usa para referirse a sucesos poco importantes y aislados en el espacio que, si se ponen en contacto, pueden provocar consecuencias trágicas.

Figura 3. La medusa negra

\begin{tabular}{|c|l|}
\hline \multicolumn{1}{|c|}{ Medusa negra } \\
\cline { 2 - 3 }
\end{tabular}

Algunas características del mundo globalizado de hoy en día, en el que la comunicación entre las personas es instantánea, presagia la frecuente aparición de medusas negras ahora y en el futuro. El ataque informático a Estonia en abril del 2007 puede ser considerado un fenómeno del tipo de las medusas negras. Se trata de un buen ejemplo de un ataque de denegación de servicio, que es 
difícil de detectar y que produjo el bloqueo de los cajeros automáticos, de las transacciones virtuales y el colapso de los servidores de las páginas web del Gobierno estonio. Pero las medusas negras no solo son frecuentes en el ámbito informático como veremos luego. Los eventos normales y aislados pueden asociarse y mutar creando situaciones extraordinarias, que pueden tener consecuencias importantes para la sociedad, la economía o la política. Por lo tanto, una medusa negra está formada por acontecimientos de poca importancia que al relacionarse de alguna manera, pueden provocar un suceso a gran escala. Como se ve, las medusas negras al igual que los otros animales referidos pueden tener un importante impacto en el mundo que nos rodea.

En zoología existen diferentes variedades de rinocerontes, pero todos ellos tienen un gran tamaño, son de tonalidades grisáceas y una vez lanzados a la carrea es mejor no encontrárselos de frente porque siempre son un gran peligro. El rinoceronte gris es una metáfora de que muchas de las cosas que salen mal fueron obvias y podrían haberse evitado tan solo enfrentándose a ellas. Y esto es tanto de aplicación en los negocios como en nuestras vidas.

Figura 4. El rinoceronte gris

\begin{tabular}{|c|l|c|}
\hline \multicolumn{1}{|c|}{ Rinoceronte gris } \\
\cline { 2 - 3 } & $\begin{array}{l}\text { 1.- Amenaza altamente probable. } \\
\text { 2.- Bien conocida e incluso obvia. } \\
\text { 3.- No se afronta porque se hace una previsión optimista de que } \\
\text { no ocurrirá. }\end{array}$ \\
\hline \multirow{2}{*}{ brexit (2016) }
\end{tabular}

Una situación que entraña riesgo se convierte en un rinoceronte gris porque no nos enfrentamos a ella. Pero no ocurre como con el elefante negro del que no se habla; del rinoceronte se habla, pero no se hace nada. ¿Cuál es el motivo? Se trata de un mecanismo psicológico de autodefensa, por el que al negar la evidencia de su existencia creemos protegernos frente a él. Por ello debemos prestar más atención a los problemas obvios. Este pudo ser el caso del brexit. Convertirse en avestruz y esconder la cabeza debajo del ala no sirve de mucho cuando un animal de dos toneladas corre dispuesto a embestirte. Rinocerontes en el ámbito del estudio de las relaciones internacionales hay de varios tipos: aquellos que ya están corriendo, los aparecen una y otra vez, los metarrinocerontes y los no rinocerontes no identificados (Carlos, 2018a, p. 6). A continuación, veremos algún concepto básico sobre la historia y las relaciones internacionales, que junto con los animales que hemos recomentado nos serán útiles para nuestro estudio.

\section{3. ¿Se repite la historia?}

No existe un concepto universal sobre qué es la Historia, pero la historia siempre se compone al menos de dos elementos: los hechos pasados y los historiadores. Por este motivo se suele considerar que la historia nunca está terminada, sino que se halla en un proceso continuo de transformación el cual no tiene fin (Carr, 1966). Además, la historia está llena de falsificaciones y de deformaciones. No se puede considerar que el alcance ético de las unas y de las otras sea diferente. En nuestra opinión aquellas "que parecen haberse realizado por los mismos prota- 
gonistas de algunos hechos fundamentales" de la historia son las peores (Caro Baroja, 1991, p. 201). Las falsificaciones son muy conocidas y a veces comentadas. El éxito de una falsedad es la demostración de que una iniciativa bien orquestada y orientada a un fin, puede cambiar la historia. No obstante, no se debe confundir la invención o fabricación de la historia, con la difusión de la historia compartida por un pueblo.

A parte de las deformaciones o las falsificaciones, los historiadores participan de diferentes escuelas historiográficas como son la escuela materialista-marxista, el positivismo histórico, la historia sociocultural o, incluso, otras corrientes más antiguas según las cuales el protagonismo de algunas personas determina la historia. Más allá de estas corrientes historiográficas nos encontramos con la creencia muy arraigada de que la historia se repite. Edward H. Carr, historiador británico y teórico de las relaciones internacionales, ya nos decía en 1961 durante unas conferencias impartidas en la Universidad de Cambridge, que la historia se repetía muy pocas veces. Sobre todo, siguiendo al profesor Carr, esto era muy poco frecuente entre las sociedades que conocían bien su propia historia; y también nos daba un motivo para que la historia no se repitiera: evitar el mismo desenlace en el futuro que hubo en el pasado, si este no era bueno para la sociedad. En nuestra opinión la historia no se repite de la misma manera; es decir, causas similares no producen las mismas consecuencias, pero sí marcan tendencias que claramente orientan el futuro. Y coincidimos con E. H. Carr en que el conocimiento histórico nos debe ayudar a preparar un futuro mejor. Pero que la historia nos pueda orientar para evitar lo malo, no excluye que si nos empeñamos podamos conseguir incluso peores resultados que en el pasado, a partir de condiciones similares. El debate de la utilidad de las ciencias sociales ya ha sido tratado con frecuencia, pero conviene recordar que las ciencias sociales deben ser ciencias para la acción, es decir, deben tener "una proyección técnica o instrumental en favor del Hombre”. Es lo que llamamos Tercera Generación de las Ciencias Sociales (Carlos, 2015, p. 4). Por eso es conveniente valerse del conocimiento histórico para así orientar nuestras acciones futuras y tratar de evitar situaciones indeseables. Es lo que denominamos Ciencias Sociales para la Acción.

\section{La sociedad internacional} es un grupo de comunidades políticas independientes, esencialmente países y organizaciones internacionales, con intereses, nomas e instituciones comunes

\section{El sistema internacional y el orden mundial}

Como hemos comentado, los atentados del 11 de septiembre de 2001 son un buen ejemplo de la aparición de un cisne negro y también al mismo tiempo son un ejemplo de un ataque contra la sociedad internacional. La crisis económica internacional de 2007 también es una buena muestra de cómo una amenaza inesperada (o un cisne negro), pueden provocar efectos devastadores en el sistema internacional. Pero veamos a que nos referimos al hablar de la sociedad internacional y que tiene que ver con el sistema internacional.

La sociedad internacional es un grupo de comunidades políticas independientes, esencialmente países y organizaciones internacionales, con intereses, normas e instituciones comunes (Bull y Watson, 1984, pp. 425-435). Hoy en día la sociedad internacional se caracteriza por tener carácter mundial, ser interdependiente y tener una regulación que está en un proceso intenso de transformación. La sociedad internacional ha creado lo que se conoce como sistema internacional. Nos referimos esencialmente al sistema de Naciones Unidas que entró progresivamente en vigor tras la Conferencia de Paz de San Francisco de 1945. Esta sociedad internacional, organizada en torno al sistema de Naciones Unidas es lo que configura un determinado orden internacional (frecuentemente asociado al final de la II Guerra Mundial), también llamado orden mundial (aunque este se suele asociar con más frecuencia al final de la Guerra Fría). El orden internacional es un 
concepto complejo y central en el estudio de las relaciones internacionales que tratan de analizar las relaciones de poder entre los diferentes actores del sistema internacional.

El orden mundial resultado de la Segunda Guerra Mundial fue un sistema bipolar de equilibrio tenso y organizado en torno a dos superpotencias y otras potencias de menor influencia. Pero este orden ya ha desaparecido, en parte como consecuencia de la desintegración de la URSS, aunque su debilitamiento fue progresivo. En 1961 el historiador Edward H. Carr ya señalaba que "después de cuatrocientos años el centro de gravedad mundial ha salido claramente de la Europa Occidental”, señalando entonces que la pauta en los asuntos mundiales la marcaba Europa Oriental y Asia (Carr, 1966, p. 200). Sin duda una visión muy temprana de la situación actual. Hoy en día tenemos un nuevo orden mundial, en el que nos encontramos con EE. UU. como superpotencia, otras potencias en ascenso como China y varias potencias regionales como por ejemplo Rusia o India. Es lo que se ha venido a denominar un sistema "unimultipolar". Estos cambios en el orden mundial han sido estudiados con frecuencia, como por ejemplo recientemente en un informe encargado por el Parlamento Europeo a Oxford Analytica. En el informe se detallan varias tendencias mundiales que auguran diferentes cambios en el orden internacional (Carlos, 2018b, pp. 544-547), así como cuál puede ser la posible evolución de algunos de sus principales actores: EE. UU., China, Rusia, la Unión Europea y el G20 (OXAN, 2017, pp. 42-46). Podemos concluir que como consecuencia de estas tendencias la hegemonía hasta ahora de Occidente cada vez será más desafiada por China, Rusia y otras potencias emergentes. Y aunque EE. UU. continuará siendo la mayor potencia militar mundial, necesitará cada vez más el apoyo de estas nuevas potencias para poder actuar. Veamos cómo será el nuevo orden mundial de la mano de los ya conocidos animales de las relaciones internacionales.

\section{Un rinoceronte embistiendo: el papel de China}

El rápido y mantenido ascenso de China en la escena internacional no puede considerarse un cisne negro, sin duda se trata de un rinoceronte a la carrera para buena parte de la comunidad internacional. Ni para India, ni para EE. UU., ni para la Unión Europea se trata de un protagonismo inesperado o improbable. En realidad, el papel cada vez más relevante de China es esperado con certeza, en parte, porque hace tiempo que se aprecian sus efectos y por la alteración que estos suponen para el funcionamiento del sistema internacional. Es de sobra conocido que el crecimiento económico de China, Brasil, Rusia, India y Sudáfrica en las dos últimas décadas ha coincidido con el estancamiento de las economías de buena parte de los países de la OCDE. El proceso de cambio acelerado mundial que es conocido como la Gran Aceleración (Steffen, Broadgate, Deutsch, Gaffney, \& Ludwig, 2015, pp. 4-7) está siendo muy bien aprovechado por China, en particular desde la llegada de Xi Jinping.

En 2013, Xi Jinping casi al mismo tiempo que ocupó la presidencia del partido, presentó en Kazajstán el proyecto One Belt, One Road (en adelante Estrategia OBOR). Los dirigentes chinos han utilizado la marca de la Ruta de la Seda de la Organización Mundial del Turismo, para dar publicidad a la Estrategia OBOR. Pero esta estrategia puesta en marcha por Jinping es un plan de expansión comercial (Lemus y Valderrey 2017, p. 61) y geoestratégico para reforzar el papel de China como potencia mundial. Como consecuencia de la crisis financiera mundial de 2008, China, desde el año 2009 vio reducidas sus exportaciones y también su producto interior bruto: el PIB pasó de estar por encima del 10\% a situarse en torno al 6,8\%. El gran reto de los gobernantes chinos fue recuperar la senda de crecimiento precedente y para ello se tomaron
El rápido y mantenido ascenso de China en la escena internacional no puede considerarse un cisne negro, sin duda se trata de un ninoceronte a la carrera para buena parte de la comunidad internacional 
dos tipos de medidas que no funcionaron: se trató de aumentar la demanda interna y también la inversión pública en infraestructuras. Ninguna de las iniciativas tuvo éxito, la primera por la propensión al ahorro de los ciudadanos chinos y la segunda por las dificultades de financiación. Por este motivo, se está tratando de recuperar el crecimiento del PIB mediante las exportaciones apoyadas por la estrategia OBOR. Pero esta estrategia va más allá: "es el mejor exponente de la ambición china de dominar el mundo llevando a cabo una política expansionista" (Carlos, 2018c, p. 122). Generalmente la estrategia OBOR es contemplada desde la perspectiva de la construcción de infraestructuras por parte de China, como ha hecho en Yibuti, en el Puerto del Pireo o al adquirir una participación mayoritaria en NOATUM, una de las principales operadoras de puertos de España (Carlos, 2018c, p. 122).

En realidad estamos ante un rinoceronte gris que está corriendo y apunto de embestir. Según Michele Wucker la forma de tratar a estos rinocerontes es enfrentarse a ellos, porque seguro que van a hacer daño (Wucker, 2016). De hecho, algunos de los acontecimientos actuales en la política internacional más importantes son consecuencia de la política comercial expansionista de China. Nos referimos a la llamada guerra comercial que parece enfrentar a EE. UU. con la Unión Europea. El dumping sostenido en el sector del acero protagonizado por China está en la base de este conflicto. Durante largo tiempo la Unión Europea en su relación con China se ha ocupado de satisfacer los intereses comerciales de China, su ingreso en la Organización Mundial del Comercio, o la transferencia de tecnología a cambio de pocas cosas: el compromiso de la defensa de las minorías étnicas en China, el refuerzo del papel de la mujer en la sociedad o la reducción de la producción del acero. China nunca se ha opuesto a las peticiones de la Unión Europea, pero nunca ha permitido avances importantes (Carlos, 2017, pp. 16, 20 y ss).

Figura 5. Un rinoceronte embistiendo

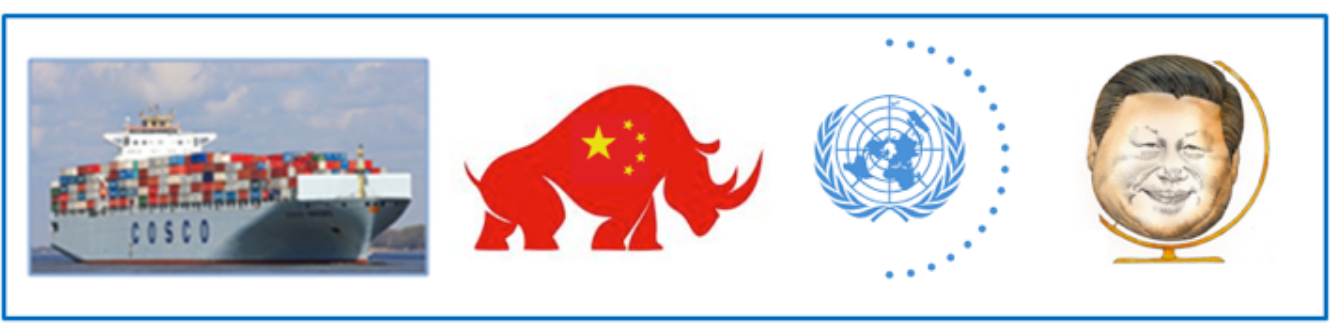

La política expansionista china es un riesgo obvio con importantes implicaciones en el orden internacional y en el sistema internacional. Esta política se ha convertido en un rinoceronte a la carrera, por la visión de una buena parte de la sociedad internacional, que ve de manera optimista la realidad, creyendo que al negar el riesgo nos protegemos ante él. Todo el mundo conoce este rinoceronte, pero además de la Unión Europea o de India pocos han tratado de prepararse ante esta nueva situación.

\section{Los elefantes: el papel de EE. UU. y de Rusia}

Como hemos comentado, un elefante negro es un posible acontecimiento de alto impacto que se encuentra más allá del ámbito de expectativas regulares, pero del que nadie habla a pesar de la evidencia existente. Veamos algunos elefantes del orden mundial actual. Si duda las 
consecuencias de los intereses estratégicos de EE. UU. y Rusia proporcionan algunos de estos elefantes. Ya hemos comentado como ejemplo de elefante la anexión de Crimea, pero también se podría referir la crisis de Ucrania o el surgimiento de la república autónoma de Abjasia (Georgia) y de Osetia del Sur, consideradas por Rusia Estados independientes. Estos elefantes dejaron de serlo, para convertirse con toda probabilidad en rinocerontes.

La idea del elefante en el análisis de las relaciones internacionales alude a un problema que es visible para todos y que tendrá enormes consecuencias pero, sin embargo nadie quiere abordarlo, ni tan siquiera hablar de él. Para la Unión Europea existe un elefante de enormes dimensiones, del que poco a poco no va quedando más remedio que reconocer su existencia y comenzar a hablar abiertamente de él: el progresivo desinterés de EE. UU. sobre los asuntos geopolíticos de importancia para Europa. Nos referimos en particular a todo lo relativo a la frontera sur de la Unión Europea y notoriamente también la frontera oriental. Ni EE. UU. ni la OTAN hasta la fecha han tomado interés por el Sahel, hasta el punto de que las diferentes iniciativas en favor de la estabilización de Mali son de Naciones Unidas, la Unión Europea o multilaterales con liderazgo francés y colaboración española entre otros países.

Figura 6. La Unión Europea tiene varios elefantes, que una vez reconocidos está gestionado bien

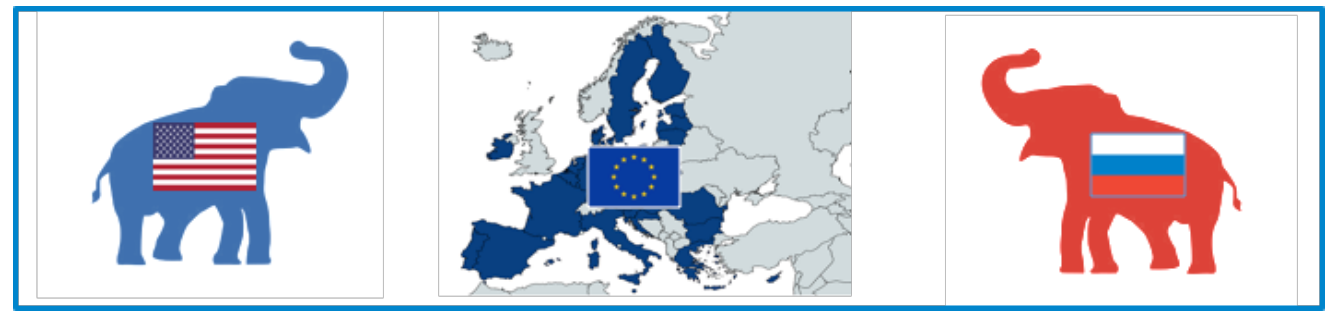

Elefantes negros podrían haber sido los que se materializaran de los intereses territoriales de la federación rusa en las repúblicas bálticas. Pero el ingreso de Estonia, Letonia y Lituania en la Unión Europea en 2004 y los posteriores acontecimientos no lo han permitido: principalmente las misiones de OTAN en esta zona. Nos referimos a la misión en Letonia llamada presencia avanzada reforzada o Enhanced Forward Presence (eFP) y la policía aérea del Báltico o Baltic Air Policing (BAP) realizada desde Estonia.

De lo que no cabe duda es de que situaciones de todos conocidas y ante las que se tardó demasiado tiempo en reaccionar, fueron el brexit o incluso la crisis migratoria del mediterráneo. Hoy en día ya no pueden ser considerados elefantes ya que lejos de ser ignorados se están tomando medidas para afrontarlos.

\section{Cisnes y la Unión Europea}

Por definición un cisne negro es un acontecimiento traumático que no se puede prever, fundamentalmente porque es de diferente naturaleza que los acontecimientos frecuentes y no va precedido de tendencias que lo anuncien. Aún así, nuestra intención es tratar de conocer los posibles cisnes e incluso medusas que pudieran afectar a la Unión Europea. Prevenirse o tratar de identificar las medusas que aún no hayan llegado, es todavía una tarea más difícil, porque se trata de eventos normales y aislados en el espacio que al conectarse pueden provocar con- 
secuencias extraordinarias. Estas consecuencias pueden ser importantes y en ocasiones muy dañinas para la sociedad, la economía o la política. Dada la imprevisibilidad de los cisnes y de las medusas, nos ayudaremos de la historia. Como sabemos la historia no se repite o, mejor dicho, las sociedades que conocen y tienen presente su historia tratan de evitar las consecuencias perniciosas de experiencias pasadas. Ahora que ya conocemos algunos elefantes y rinocerontes que pueden afectar a la Unión Europea, veamos si somos capaces de identificar algún cisne o medusa con la ayuda de la historia.

Para algunos autores la Primera Guerra Mundial fue más bien una guerra civil europea con consecuencias mundiales (Carr, 1964, p. 201). Pero buena parte de los historiadores coinciden en que su inicio fue consecuencia de múltiples factores como, por ejemplo, el enfrentamiento francoalemán, la tensión entre el Reino Unido y Alemania por el control marítimo o la tensión en los Balcanes. En realidad, el sistema ideado por Bismarck, que duró más de 20 años, solo aplazó las tensiones del este de Europa, creadas principalmente por el desmoronamiento del Imperio Otomano. El equilibrio tenso mantenido durante la era Bismarck desapareció bruscamente y comenzó lo que se ha denominado la Paz Armada (1890-1914), que son los años previos a la Primera Guerra Mundial. No se puede culpar únicamente al canciller Otto Von Bismarck de lacerar los problemas y no afrontarlos (Carr, 1964, p. 175). Pero la realidad es que el sistema de alianzas establecido entre 1871 y 1893 trató el interés de las distintas potencias sobre el este de Europa como si fueran varios rinocerontes, que al final se llevaron a la carrera el inestable orden internacional establecido a partir del sistema de alianzas. La Paz Armada se rompió por la aparición de un cisne negro: el asesinato del archiduque heredero de Imperio Austrohúngaro en Sarajevo. En realidad, este cisne fue producido por lo que hoy llamamos una medusa. La medusa que apareció durante la Paz Armada estuvo constituida por acontecimientos normales y separados en el tiempo y el espacio. Entre ellos caben destacar los siguientes: la segunda revolución industrial, el avance progresivo del colonialismo, la carrera de armamentos o las tensiones entre Rusia y el Imperio Austrohúngaro en el este de Europa.

Ciertamente la historia no se repite, ni tan siquiera circunstancias parecidas, acompañadas de acciones similares, producen los mismos resultados. Pero nuestro análisis debe entender qué diferencias y similitudes hay entre el pasado y el presente y que circunstancias actuales o próximas pueden producir la aparición de alguno de nuestros animales. En realidad, se trata de usar las metáforas de las relaciones internacionales para ayudarnos a prevenirnos de aquello que pueda pasar y no convenga demasiado. Imaginar que un magnicidio, como el que ocurrió en 1914 en Sarajevo, podría ser el próximo cisne negro no conduce a ningún sitio. Pero el panorama del abastecimiento de la energía en Europa es muy paradigmático de todo lo comentado hasta ahora y en nuestra opinión tiene importantes similitudes con el sistema de alianzas de la era Bismarck.

La Comisión Francesa de la Regulación de la Energía ha señalado hasta siete regiones energéticas en Europa y tres regiones en relación al gas (Carlos, 2016, pp. 561-563). Cada una de ellas tiene diferentes intereses y necesidades energéticas. Por este motivo, hay distintas iniciativas por parte de los Estados miembros de la UE que tratan de defender sus intereses particulares. Así Alemania se encuentra presente en cuatro de estas iniciativas, que son las siguientes: el Foro Quíntuple de la Energía, el Grupo Bake, la Red Gasista de los Mares del Norte y el BEMIP. El Grupo Baake liderado por Alemania agrupa a los países del Foro Quíntuple de la Energía y a otros 5 países. Mientras tanto la iniciativa para el desarrollo de la Red Gasista de los Mares del Norte está constituida por diez Estados interesados en el desarrollo de un gaseoducto
La realidad es que el sistema de alianzas establecido entre 1871 y 1893 trató el interés de las distintas potencias sobre el este de Europa como si fueran varios rinocerontes 
por el norte como alternativa al abastecimiento continental. Además, Alemania también está presente en una cuarta iniciativa lanzada por Comisión Europea en 2008 para la Interconexión del Mercado Báltico Energético (Baltic Energy Market Interconnection Plan o BEMIP).

Todas estas iniciativas están orientadas a asegurar el suministro de gas proveniente de Rusia, pero en unas ocasiones junto con unos países y en otras ocasiones con otros. Algo muy normal para conseguir asegurar la defensa de los intereses energéticos de Alemania: que hasta ahora ha sido el suministro desde Rusia. De hecho, la canciller alemana Ángela Merkel ha mantenido diversas reuniones con Putin, en particular en relación con la crisis de Ucrania y el aseguramiento del suministro de gas a Alemania. Aunque es más inquietante la reunión mantenida en julio de 2018 en la que Merkel se reunió en Berlín con el general ruso Valery Gerasimov, jefe del Estado Mayor y fundador de la llamada "doctrina Gerasimov", sobre la guerra híbrida. Al mismo tiempo en el Libro Blanco de la Defensa de Alemania publicado en julio de 2016 se muestra la voluntad alemana de ocupar un papel más central en la OTAN y en la Política de Seguridad y Defensa de la Unión. De hecho, Alemania está haciendo todo lo que está a su alcance para asegurar el suministro y eso no siempre coincide con los intereses de quienes no son ni Rusia, ni Alemania. La canciller alemana al igual que Bismarck necesita que el este de Europa esté en paz y para ello está tratando de establecer pactos que no favorecen la integración de la UE, ni solucionan los conflictos. Estos pactos están orientados a establecer diferentes gasoductos que eviten las zonas en conflicto. Pero existen otras alternativas como es el abastecimiento desde Argelia a través de España y Francia. Pero para ello se debe contar con la aprobación francesa, que no se opone, pero no facilita la llegada de este gas porque supone una competencia para su energía eléctrica de origen nuclear.

Nada de lo comentado hasta ahora nos permite identificar cisnes o medusas, pero sí un panorama complejo y no completamente desconocido que se puede resumir en que Alemania y EE. UU. siguen interesados en lograr al menos la estabilidad formal del este de la UE, casi a cualquier precio. Pero la realidad es que existe una situación de tensión acumulada en el este de la UE como consecuencia de la desintegración de la República Federal Socialista de Yugoslavia en 1992, de la política expansionista rusa en Ucrania, de la crisis migratoria en Grecia de 2015 o de la crisis económica de 2007, entre otros factores. Considerar que un magnicidio, como ocurrió en 1914 en Sarajevo, podría ser el próximo cisne negro de la Unión Europea no conduce a ningún sitio, pero si debemos estar atentos a los posibles acontecimientos aislados que no correspondan a las tendencias conocidas.

\section{Las medusas y el sistema internacional}

Los acontecimientos separados geográficamente, en apariencia de escasa importancia, al coincidir en el tiempo o al ponerse en contacto de alguna manera, pueden provocar unas consecuencias de gran importancia y de distinta naturaleza que pueden generar mucha inestabilidad. Este tipo de acontecimientos son las ya conocidas medusas negras. Por lo tanto, conviene estar atentos a acontecimientos localizados en cualquier parte del mundo que, aun siendo de poca importancia, puedan anunciar la formación de un animal de este tipo.

Una medusa negra de colosales dimensiones y catastróficas consecuencias podría estar formándose y atentar contra el sistema internacional actual. Como hemos comentado el sistema internacional ha sido creado por la sociedad internacional que en 1945 esencialmente estaba constituida por los Estados y por las organizaciones internacionales. Pero hoy en día a la socie-
Considerar que un magnicidio, como ocurrió en 1914 en Sarajevo, podría ser el próximo cisne negro de la Unión Europea no conduce a ningún sitio 
dad internacional se han venido incorporando progresivamente otros actores con diferentes derechos y deberes como son las personas, las grandes empresas globales e incluso otras entidades no estatales como las organizaciones no gubernamentales. El resultado es que el derecho internacional público que regula el sistema internacional ha incorporado solo algunas modificaciones, ya que los cambios han sido tan rápidos que el derecho internacional no ha podido recoger la situación actual.

El proceso de debate intenso desarrolló en el siglo pasado un marco jurídico adaptado a las nuevas circunstancias resultado de la Segunda Guerra Mundial, pero ahora este proceso se encuentra prácticamente interrumpido. Naciones Unidas ha avanzado muchísimo, pero hoy en día más que nunca se enfrenta, en nuestra opinión, a un enorme reto: adaptar su organización y su funcionamiento a diferentes patrones culturales. Aunque parezca contradictorio, la globalización propia del siglo XXI asociada a una cultura tecnológica común, avanza al mismo tiempo por un camino de afirmación de la "diversidad cultural". De hecho, el debilitamiento del sistema de Naciones Unidas (Izaguirre, 2018. p. 35) es consecuencia de esta diversidad cultural. Diversidad que cuestiona de facto los derechos universales, en particular los protocolos sobre los derechos civiles y políticos, así como el de los derechos económicos, sociales y culturales. El motivo es que la progresiva importancia en el orden internacional de actores diferentes a los estados y organizaciones internacionales, así como el mayor protagonismo de algunas potencias en el orden mundial como China. Desde su fundación Naciones Unidas ha tratado de dar cabida a las diferencias culturales, pero lo ha hecho atendiendo a un criterio geográfico, organizando diferentes grupos regionales. Por ejemplo, hoy en día el Consejo Económico y Social (ECOSOC) está agrupado regionalmente en cinco áreas geográficas que son las siguientes: África, América Latina y Caribe, Asia Pacífico, Europa Occidental, Europa Oriental y otros miembros independientes como EE. UU. No obstante, este criterio regional no coincide completamente con las diferencias culturales y no puede constituirse en un elemento que permita renovar, actualizar y regenerar la institución. Seguramente un criterio de áreas culturales frente al propiamente geográfico, en algunos de los instrumentos de Naciones Unidas fortalecería el sistema.

Pero antes comentábamos que la creación de una medusa negra de grandes dimensiones y formada poco a poco, podría hacer quebrar el sistema internacional fundamentado en Naciones Unidas y en el derecho internacional público. Algunos de los elementos que podrían participar en la creación de esta gran medusa podrían estar relacionados con los siguientes hechos: (1) áreas geográficas controladas por actores no estatales, (2) sistemática violación del derecho internacional público y del derecho internacional humanitario y (3), políticas comerciales proteccionistas y a la vez expansionistas. Entre las áreas geográficas controladas por actores no estatales frecuentemente se suelen referir los estados fallidos o parte de ellos como ocurre en el Sahel, en Transnistria, Abjasia, Osetia de Sur o Nagorno Karabaj entre otros lugares. Como violación del derecho internacional público y del derecho internacional humanitario también se pueden referir hechos de la naturaleza de la crisis humanitaria de los Rohingyas o la confusión en la crisis migratoria del Mediterráneo de los refugiados, los inmigrantes irregulares y el deber de socorro en el mar. También parecen cada vez más importantes en la formación de esta medusa, las políticas comerciales proteccionistas y al mismo tiempo expansionistas que se están poniendo en práctica. Nos referimos en particular al posible abandono de EE. UU. de la Organización Mundial del Comercio y al papel jugado por China en esta institución, ya que siempre EE. UU. y la Unión Europea acusan a China de no respetar la economía de mercado.
Parecen cada vez más importantes en la formación de esta medusa, las políticas comerciales proteccionistas y expansionistas que se están poniendo en práctica 
De hecho, la actividad internacional de China bordea sistemáticamente (si no es que lo vulnera) el derecho internacional público y así como los sistemas jurídicos nacionales. El motivo es que allá donde realiza sus operaciones en el exterior, a través de empresas de naturaleza jurídica privada, pero de carácter estatal, trata de evitar la legislación local y aplicar la de su propio país. De hecho, China de manera silenciosa está tratando de cambiar de facto las normas de gobernanza mundial, es decir el sistema internacional

La estrategia china One Belt, One Road o estrategia OBOR es un buen exponente de la voluntad de evitar las normas de gobernanza mundiales. Esta misma estrategia se ha valido de una iniciativa de Naciones Unidas (la Ruta de la Seda de la UNESCO), apropiándose del término Ruta de la Seda para su expansión comercial y geoestratégica.

\section{Conclusión: icuidado con las medusas negras!}

En los últimos años se han empleado algunos animales como metáfora para entender mejor los conflictos del mundo actual: son los animales de las relaciones internacionales. Estos animales imaginarios nos permiten reflexionar sobre los cambios que están afectando al mundo y sobre todo anticiparnos a algunos acontecimientos y situaciones que están por llegar.

Para acercarnos mejor a estos cambios además estos animales metafóricos hemos revisado algunos conceptos básicos sobre la historia y las relaciones internacionales. Las ciencias sociales de tercera generación son aquellas que tienen una utilidad práctica en favor del hombre. Es lo que denominamos ciencias sociales para la acción. Por ello el conocimiento de la historia, junto con el estudio de las relaciones internacionales debe servirnos para orientar nuestras acciones futuras y tratar de evitar situaciones indeseables. Las relaciones internacionales se esfuerzan por conocer el orden internacional que es el resultado de la interacción de la sociedad internacional, que en su devenir crea un sistema de relaciones que denominamos sistema internacional. El sistema internacional actual se fundamenta en el sistema de Naciones Unidas, que fue resultado de la conferencia de San Francisco tras la Segunda Guerra Mundial. En los últimos años este sistema se está debilitando como consecuencia de los cambios acaecidos desde finales del siglo pasado y por la tendencia al bilateralismo en las relaciones internacionales propia de nuestros días. El sistema internacional hoy corre el peligro de ser sustituido tácitamente por un nuevo sistema ad hoc, que perjudica la estabilidad mundial y en particular a la Unión Europea.

Nuestros animales nos proporcionan marcos explicativos que son muy útiles en el proceso de cambio continúo y acelerado motivado por la globalización. Gracias a ellos, podemos analizar mejor el mundo en que vivimos y tratar de comprender algunas situaciones y crisis internacionales que son difíciles de prever. Hemos visto que un cisne negro es una amenaza altamente improbable, pero que puede tener efectos devastadores. Un elefante negro es un problema bien conocido y de importantes consecuencias del que no se habla y nadie quiere abordar. Una medusa negra alude a sucesos de poca importancia, que al conectarse pueden un provocar un acontecimiento de gran importancia. Por último, un rinoceronte gris es una amenaza altamente probable y de gran impacto que es conocida pero que no se afronta.

El rápido y mantenido ascenso de China en la escena internacional es un rinoceronte a la carrera para buena parte de la comunidad internacional. Desde la llegada de Xi Jinping China ha lanzado una ofensiva comercial mundial parar tratar de recuperar el ritmo de crecimiento interrumpido en el año 2007, es la estrategia OBOR. Esta estrategia le ha llevado a la cons- 
trucción de infraestructuras en Yibuti, en el Puerto del Pireo o a la adquisición de una participación mayoritaria en NOATUM, una de las principales operadoras de puertos de España. La estrategia OBOR coincide con una fuerte inversión en su marina de guerra, que quiere controlar militarmente el Índico y el mar de China meridional en los próximos años. La política expansionista china atenta contra los intereses de múltiples actores y al mismo tiempo es una amenaza para el sistema internacional. Todo el mundo conoce este rinoceronte, pero además de la Unión Europea o de India pocos países han tratado de prepararse ante esta nueva situación.

Un elefante negro es un acontecimiento que probablemente ocurra y que tendrá alto impacto, pero que es ignorado a pesar de su innegable existencia. Algunos elefantes como la anexión de Crimea o la crisis de Ucrania dejaron de serlo convirtiéndose en rinocerontes. Para la Unión Europea existe un elefante de enormes dimensiones: el progresivo desinterés por parte de EE. UU. sobre los asuntos geopolíticos de importancia para Europa. Nos referimos en particular a todo lo relativo a la frontera sur de la Unión Europea y notoriamente también la frontera oriental. Ni EE. UU. ni la OTAN hasta la fecha han tomado interés por el Sahel, hasta el punto de que las diferentes iniciativas en favor de la estabilización de Mali son de Naciones Unidas, la Unión Europea o multilaterales con liderazgo de Francia y colaboración española entre otros países.

Por definición un cisne negro es un acontecimiento traumático que no se puede prever. No obstante, hemos tratado de identificar cuáles podrían ser las consecuencias para la Unión Europea de la aparición de un suceso traumático de este tipo, o incluso de la aparición de alguna medusa. Estas últimas todavía son más difíciles de anticipar, porque las medusas se forman al ponerse en contacto eventos normales provocando consecuencias extraordinarias. Para todo ello nos hemos servido de la historia y hemos establecido la comparación entre la Europa liderada por las alianzas de Bismarck, comparándola los pactos establecidos hoy en día para el abastecimiento energético de los Estados europeos. La historia no se repite, pero nos debe orientar la acción del futuro. No hemos conseguido identificar ningún cisne o medusa que amenace la Unión Europea, porque por definición son inesperados. Pero debemos estar atentos ante posibles acontecimientos aislados y en apariencia normales que no correspondan con las tendencias conocidas, sus consecuencias podrían ser catastróficas.

Creemos que se está formando una medusa negra de dimensiones descomunales y que atenta contra el sistema internacional actual fundamentado en Naciones Unidas y en el derecho internacional público. Algunos de los elementos que podrían llegar a crear esta medusa son: algunas áreas geográficas controladas por actores no estatales, una sistemática violación del derecho internacional público y del derecho internacional humanitario y por último las políticas comerciales proteccionistas y a la vez expansionistas de algunas potencias como EE. UU., Rusia y en particular de China. China sin oponerse a casi nada en la escena internacional está tratando de cambiar las normas de gobernanza mundial.

En nuestra opinión, un elemento que podría evitar o al menos aplazar la formación de esta medusa, es el fortalecimiento del sistema de Naciones Unidas mediante la incorporación de áreas culturales, inicialmente en convivencia con los grupos regionales ya existentes, como los del Consejo Económico y Social de Naciones Unidas. Este cambio puede animar la actualización y renovación de Naciones Unidas y del proceso de codificación del derecho internacional. 


\section{Referencias}

Bull, H., \& Watson, A. (1984). The expansion of international society. Clarendon Press: Oxford University Press.

Caro Baroja, J. (1991). Las Falsificaciones de la Historia (En relación con la de España). Barcelona: Círculo de Lectores.

Carlos Izquierdo, J. de (2015). Enfoque Cultural y Relaciones Internacionales. El caso de El Salvador. Revista Electrónica Iberoamericana, 9(1), 1-19.

Carlos Izquierdo, J. de (2016). La estrategia de seguridad energética de la Unión Europea y España. BIE 3: Boletín IEEE, (1), 557-574. DOI: https://doi.org/10.5209/runi.53788

Carlos Izquierdo, J. de (2017). Las relaciones bilaterales entre China y la Unión Europea. BIE 3: Boletín IEEE, (8), 947-969. Recuperado de https://goo.gl/pQvq96.

Carlos Izquierdo, J. de (2018a). Cisnes, elefantes, medusas y rinocerontes. Las Relaciones Internacionales y sus animales. Comillas Journal of International Relations (12), 1-8. DOI: https://doi.org/10.14422/cir.i12.y2018.001

Carlos Izquierdo, J. de (2018b). Horizonte 2035. OTAN, Unión Europea y España. BIE: Boletin IEEE, (9), 542-565. Recuperado de https://goo.gl/SCDksL.

Carlos, J. de (2018c). La Estrategia Global de China: Dominar el Mundo desde la Mar. Revista General de Marina, (275), 115-123. Recuperado de https://goo.gl/QasvSc

Carr, E. H. (1964). What is History? The George Macaulay Trevelyan Lectures Delivered in the University of Cambridge January-March 1961. Barcelona: Seix Barral.

Lemus, D., y Valderrey, F. (2017). La Nueva Ruta de la Seda y la Diplomacia Internacional de los negocios. Comillas Journal of International Relations, (10), 47-64. DOI: https://doi. org/10.14422/cir.i10.y2017.004

Izaguirre, J. L. (2018). Organizaciones internacionales. Comunicar la utilidad. Comillas Journal of International Relations, (12), 28-37. DOI: https://doi.org/10.14422/cir.12.y2018.003

OXAN, Oxford Analytica (2017). Global Trends to 2035. Geo-politics and international power. European Parliamentary Research Service. Global Trends Unit.

Steffen, W., Broadgate, W., Deutsch, L., Gaffney, O., \& Ludwig, C. (2015). The trajectory of the Anthropocene: The Great Acceleration. The Anthropocene Review, 2(1), 81-98. DOI: https://doi.org/10.1177/2053019614564785

Taleb, N. N. (2007). The Black Swan. The Impact of the Highly Improbable. New York: Random House. Reviewed by James Iain Gow. Canada: Université de Montréal.

Wucker, M. (2016). The gray rhino: How to recognize and act on the obvious dangers we ignore. Macmillan. 